\title{
Factors Correlated with Pre-Exposure Prophylaxis (PrEP) Awareness and Use among Black Adults in the United States: Implications for Improving PrEP Uptake
}

Kennethea A. Wilson ( $\sim$ kaw5776@buffalo.edu )

University at Buffalo - The State University of New York https://orcid.org/0000-0003-3404-2607

Sarahmona M. Przybyla

University at Buffalo School of Public Health and Health Professions

Jacob Bleasdale

University at Buffalo School of Public Health and Health Professions

\section{Steven Gabriel}

University at Buffalo School of Public Health and Health Professions

Natalie Leblanc

University of Rochester School of Nursing

Noelle St. Vil

University at Buffalo School of Social Work

\section{Research Article}

Keywords: pre-exposure prophylaxis (PrEP) awareness, Black adults

Posted Date: April 14th, 2021

DOI: https://doi.org/10.21203/rs.3.rs-345917/v1

License: (c) (i) This work is licensed under a Creative Commons Attribution 4.0 International License. Read Full License 


\section{Abstract}

In the United States, low rates of pre-exposure prophylaxis (PrEP) awareness and utilization persist among Black adults. To date, few studies have assessed PrEP awareness and use among a nationally representative sample of Black adults. The present study explored factors associated with PrEP awareness and use among Black adults in the United States. Most participants were unaware of PrEP (71\%). Those reporting a history of incarceration [aOR $1.73(1.04,2.90), p<.05]$ and lifetime sexually transmitted infection testing [aOR $1.76(1.19,2.59), p<.05]$ had greater odds of awareness. In the sample, only $6 \%$ of participants had ever taken PrEP. Those with a history of incarceration [aOR 9.96 (2.82, 35.14), p <.05], concurrent sexual partners [aOR $1.09(1.00,1.18), p<.05$ ], and substance use during sex [aOR $4.23(1.02,17.48), p<.05]$ had greater odds of use. Interventions to improve PrEP uptake among Black adults must consider the contextual factors associated with awareness and use.

\section{Introduction}

HIV transmission remains a significant public health concern for Black persons in the United States despite substantial scientific advancements in testing, treatment, and care. Recent national estimates indicate that 37,968 Americans were diagnosed with HIV in 2018 (1). Among those diagnosed, nearly half (42\%) were among Black/African Americans (Black) (1). Black persons (i.e., individuals with ancestral origins from Africa) account for approximately $13 \%$ of the national population (2). However, they are disproportionately affected by the HIV epidemic, regardless of sex, sexual orientation and gender expression (3). There are specific Black groups with higher lifetime risks of HIV diagnosis relative to their White counterparts, including: 1 in 2 among men who have sex with men (MSM) (vs. 1 in 11), 1 in 20 among men (vs. 1 in 132) and 1 in 48 among women (vs. 1 in 880) (4). Disparities exist in knowledge of one's HIV status as well; of the 161,800 people who were unaware of their HIV status, 67,800 (42\%) were Black (1). This disparity exists despite the fact that Blacks engage in HIV testing at higher rates than other U.S. ethno-racial groups (5).

The disproportionate incidence and prevalence of HIV infection among Black persons in the United States have been driven by significant challenges in preventing future infections (6). Many of these challenges are related to antiBlack racism and stigma, HIV stigma, homophobia, and poverty $(6,7)$. Additionally, low knowledge of HIV status and higher rates of sexually transmitted infections (STIs; most notably chlamydia, gonorrhea and syphilis) among Black persons have further curbed prevention efforts $(6,8)$. Historically, HIV prevention research has primarily focused on White MSM and overlooked diverse socio-cultural contexts integral to overcoming prevention challenges in other susceptible groups $(9,10)$. These issues have adversely impacted the behaviors, risk perceptions, and trust in the health care system among many Black Americans (10). Understanding the contextual factors that enhance adoption of HIV prevention may assist with addressing HIV-related disparities.

An effective HIV prevention strategy is the use of pre-exposure prophylaxis (PrEP) (11). PrEP is a once-daily, oral medication prescribed to HIV-negative individuals to prevent HIV acquisition (12). Eligible candidates for PrEP have known HIV risk behavior such as engaging in condomless anal and/or penile-vaginal sex, and injection drug use (13). In response to the HIV epidemic, the federal government implemented a strategic initiative to prevent new HIV infections by $90 \%$ by 2030 through the use of proven intervention strategies, such as PrEP (14).

Due to targeted uptake, not all populations have benefitted equally from $\operatorname{PrEP}(15,16)$. Using national surveillance data, Smith et al. estimated that among the 1.1 million American adults who were eligible to take PrEP in 2015, nearly half $(500,340)$ were Black (17); however, only 4,317 (11.2\%) Black adults had ever used PrEP (18). Research

Loading [MathJax]/jax/output/CommonHTML/jax.js e in PrEP coverage among Black persons, particularly among MSM, 
would decrease the racial disparity gap in HIV incidence by at least 50\% (19). The limited use of PrEP among Black adults may be explained by multifaceted factors such as low awareness (20), low HIV risk perception (21-23), HIVrelated stigma $(21,24,25)$, health care provider biases $(26)$, distrust for medical systems $(23,25)$, and medicationrelated concerns such as side effects and cost $(22,25,26)$. These barriers have hindered efforts to improve PrEP utilization within the population. Given this gap, future work must seek to identify the factors that promote awareness and use among those who could benefit from PrEP.

Much of the research on PrEP awareness and use among Black adults highlights differences by sex and sexual orientation. For example, Kanny et al. found that Black MSM were significantly less likely than White MSM to be aware of PrEP, discuss PrEP with a health care provider, or use PrEP in the past year (16). While there are some studies exploring PrEP awareness and interest among Black women $(20,25,27)$ there is limited research assessing PrEP use among this key population. Nationally, PrEP use among women regardless of race exhibits a gross disparity (18); yet, evidence indicates that relative to their White counterparts Black women are most affected. Longitudinal data from 2016 found that among a total of 78,360 PrEP users in the United States, 1,146 were female users with nearly half (48.3\%) identifying as White and only 297 (25.9\%) reported as Black (18). Similarly, Johnson et al. found low levels of PrEP awareness and knowledge among Black women recruited from a family planning clinic despite evidence of HIV risk behaviors, such as condomless vaginal sex and STI diagnoses (20). Although PrEP is a promising prevention tool for HIV prevention (28), its population-level impact on Black adults has not been fully realized.

To fully achieve PrEP equity in awareness and use, research must further explore factors and determinants extending beyond racial identity that may influence diverse pathways towards uptake. Currently, scare information is known about the correlates of PrEP awareness and use among Black adults. Key literature examining the correlates of PrEP awareness and use largely focuses on Black men and transgender women who have sex with men while excluding heterosexual Black men and women $(29,30)$. The purpose of the present study was to assess the factors correlated with PrEP awareness and use in a nationally representative sample of Black adults in the United States.

\section{Methods}

\section{Study Sample and Procedures}

Comprehensive details regarding the study sample and procedures can be found elsewhere (31). In brief, we contracted with Qualtrics $@$, an online survey platform, to recruit a nationally representative sample of Black adults with regards to income and gender in May 2018. The eligibility criteria included the following: self-identification as Black/African American, 18-40 years old, and in an intimate relationship within the past two years. The final study sample consisted of 1,050 adults. Participants who met the study's inclusion criteria were sent an email invitation to complete an online survey. Eligible participants were informed of the study procedures and then documented consent prior to survey initiation. Upon survey completion (approximately 20 minutes), participants received \$10 compensation. All study procedures were approved by the [BLINDED] Institutional Review Board.

\section{Measures}

Sociodemographic information. We collected self-report data on participants' age, gender, sexual orientation, educational attainment, annual household income and incarceration history. 
Sexual history and sexual risk behaviors. We also asked participants a variety of sexual health- and risk behaviorrelated questions, including relationship status, number of current and lifetime sex partners, lifetime STI testing and diagnosis, and substance use during sex. We asked participants (yes/no) if they considered themselves to be in a monogamous relationship and to self-report the number of current and lifetime sex partners with an open-ended response. We operationalized number of current sex partners as concurrent sexual partners for responses greater than 1. We also asked participants if they have ever tested for STIs in their lifetime (i.e., lifetime STI testing, yes/no) and if they had been diagnosed with an STI in their lifetime (i.e., lifetime STI diagnosis, yes/no). Lastly, we asked participants (yes/no) if they engaged in substance use (e.g., alcohol, marijuana) during sex.

PrEP awareness and lifetime use. We first assessed whether participants were aware of PrEP ["Before today, had you ever heard of the pre-exposure prophylaxis, also known as PrEP?']. Black adults who reported affirmatively were considered PrEP aware. We then asked PrEP-aware participants about PrEP use. We operationalized lifetime PrEP use as affirmative responses to current or past use (i.e. "I currently take PrEP" and "I have taken PrEP in the past, but do not take it now').

\section{Statistical Analysis}

In the present study, only participants who responded to PrEP questions were analyzed $(\mathrm{N}=666)$. Our data analytic plan occurred in three phases. First, we conducted a descriptive analysis on all variables of interest, including examinations of means, standard deviations and ranges for continuous variables and frequencies for categorical variables. Second, we conducted bivariate analyses (Chi-square and t-tests) to understand the relationship between participant characteristics and PrEP awareness at $\alpha$-level $=0.05$. We then constructed a multivariable logistic regression model to quantify the relationship between PrEP awareness and key covariates. Covariates were selected based on the results of independent Chi-square and t-tests. PrEP awareness was significantly associated with income $(p<0.05)$, monogamous relationship status $(p<0.05)$, incarceration history $(p<0.001)$, substance use during sex $(p<0.01)$ and lifetime testing for STIs $(p<0.001)$. We included sexual orientation as an additional covariate in our model due to its trend-level association with PrEP awareness $(p<0.09)$. Adjusted odds ratios (aOR) and 95\% confidence intervals were calculated to quantify the relationships between significant covariates and the outcome of interest (PrEP awareness).

Lastly, to understand the relationship between participant characteristics and PrEP use, we conducted bivariate analyses (Chi-square and t-tests). Next, we constructed a multivariable logistic regression model to quantify the relationship between PrEP use and key covariates. The selected covariates were based on the results of independent Chi-square and t-tests. PrEP use was significantly associated with gender $(p<0.05)$, age $(p<0.05)$, incarceration history $(p<0.001)$, and concurrent sexual partners $(p<0.05)$. As such, we included these variables as covariates the multivariable logistic model. Due to trend-level associations with PrEP use, we included lifetime STI diagnosis ( $p<$ $0.06)$ and substance use during sex $(p<0.06)$ as additional covariates in our model. We calculated adjusted odds ratios (aOR) and 95\% confidence intervals to quantify the relationships between significant covariates and the outcome of interest (PrEP use). We conducted all statistical analyses using STATA Version 16 (College Station, TX).

\section{Results}

Of the 666 participants, the majority were female (69\%), identified as African American (91\%), heterosexual (85\%) and between the ages of 18 and 29 (53\%). Nearly three-fourths of the sample indicated being in a monogamous relationship. With regards to sexual health, $61 \%$ of participants had ever tested for an STI with more than one-third 
(39\%) reporting an STI diagnosis in their lifetime. Greater than half of the sample (59\%) reported substance use (e.g., alcohol, marijuana) during sex. Additional participant characteristics appear in Table 1.

The majority of the sample was unaware of PrEP (71\%). Table 2 reports the first multivariable logistic regression model. Participants who reported a history of incarceration (aOR 1.73; 95\% Cl 1.84, 2.89) and lifetime STI testing (aOR $1.76 ; 95 \% \mathrm{Cl} 1.19,2.59)$ had significantly greater odds of being aware of PrEP. There were no significant differences in PrEP awareness by income, monogamous relationship status, sexual orientation and substance use during sex.

Of those who were PrEP aware, 20\% were currently taking or had previously taken PrEP. Results from Table 3 presents the findings from our second multivariable logistic regression model. Findings indicate that participants with a history of incarceration (aOR 9.96; 95\% Cl 2.82,35.14), engagement in substance use during sex (aOR 4.23; $95 \% \mathrm{Cl} 1.02,17.48)$ and a greater number of current sex partners $(\mathrm{aOR} 1.09 ; 95 \% \mathrm{Cl} 1.00,1.18)$ had significantly greater odds of PrEP use (See Table 3). Among PrEP users, there were no significant differences by age, education, gender, and lifetime STI diagnosis.

\section{Discussion}

Considering that Black adults have the highest estimated indications for PrEP in the United States (17), low rates of PrEP awareness and utilization are of notable concern. Overall, we found low reported rates of PrEP awareness (29\%) and lifetime use (6\%) of PrEP among Black adults. Our findings were in range with other population-specific cross-sectional studies demonstrating low levels of PrEP awareness (e.g., 10\% in Friedman et al. and 39\% in Eaton et al.) and PrEP use (e.g., $4.6 \%$ in Eaton et al. and 8\% in Friedman et al.) in the population $(29,30)$. Although gender was not a significant correlate of PrEP awareness, the predominance of Black heterosexual women in this sample may partially explain the overall limited awareness and use, given that PrEP uptake research among Black persons has historically emphasized males $(15,20)$. The current study is among the first to assess multiple factors correlated with PrEP awareness and use among Black adults in the United States. Our findings expand upon the extant literature and offer critical insights into the contextual significance of various correlates related to PrEP awareness and use.

Despite a low awareness of PrEP among Black adults, the current study found that individuals with histories of incarceration and STI testing were more likely to have heard of PrEP. Earlier cross-sectional studies have indicated associations among similar factors related to PrEP awareness. For example, Friedman et al. found gay community support, polydrug use, and past STI diagnosis as correlates of PrEP awareness among black men who have sex with men and women (29). Eaton et al. demonstrated being in a relationship, HIV testing, and others' awareness of sexuality were associated with PrEP awareness among Black men and transgender women $(29,30)$. In this study, we found that lifetime reports of incarceration and STI testing were strong predictors of PrEP awareness. Consistently, research suggests that structural levels factors may influence PrEP awareness among Black adults in the United States $(28,32)$.

Given the strong correlation between PrEP awareness with histories of incarceration and STI testing, it is possible that PrEP-aware individuals may have benefited from their involvement in criminal justice systems and accessing sexual health clinics. In particular, the periods of incarceration and post-release have been shown to increase the potential risk of HIV and STI acquisition (33-35). In the present study, one in three Black adults had ever been STI diagnosed compared to one in five nationally (36). Alongside efforts to prevent HIV, urgent attention from public

Loading [MathJax]/jax/output/CommonHTML/jax.js ress the co-occurring burden of STIs among Black adults in the United

Page 5/13 
States. The National Commission on Correctional Health Care (NCCHC) recognizes the high prevalence of some STIs (i.e., chlamydia, gonorrhea, syphilis) and substance use disorders among persons entering jails, prisons, and juvenile facilities (37), especially among Black persons (38). In response to this public health concern, the NCCHC has recommended that upon entry, HIV-negative persons who test positive for STIs are informed about PrEP (37). Much of the empirical evidence assessing the impact of interventions for HIV, STI and substance use among Black adults with criminal justice involvement (CJI) have emphasized sexual health screenings and education, which has demonstrated increases in HIV testing, knowledge, and condom-intentions (34). However, a gap remains concerning the impact of PrEP for HIV prevention on Black adults with CJI. Previous research $(24,39,40)$ has shown that PrEP awareness and acceptability is shaped by individual, social and sexual network, and community level factors, which in turn, should be leveraged to promote PrEP uptake and adherence among CJI adults. Given the emerging literature, innovative strategies and interventions designed to implement PrEP must consider the relevant factors influencing PrEP awareness. Specifically, study findings underscore the significance of various approaches that link adults with incarceration history and HIV risk to comprehensive programs facilitating PrEP access $(33,41,42)$. For example, in people with CJI and HIV risk, Peterson et al. found low PrEP awareness and HIV risk perception; yet demonstrated that a program for addiction treatment medication offered by a correctional facility served as a useful pathway to increase PrEP information and may also bolster access and retention upon release (42). It is likely that Black adults with histories of incarceration who report HIV risk behaviors would benefit from receiving tailored PrEP communication, including from their social networks and community providers (43). Enhanced STI screenings and PrEP-related messaging provided by correctional institutions may help to mitigate HIV and STI disease transmission in community settings.

We observed several factors correlated with PrEP use. Overall, history of incarceration, concurrent sexual partners, and substance use during sex were associated with PrEP use among Black adults. History of incarceration remained a strong predictor of PrEP use, indicating the significance of this correlation as it relates to PrEP uptake. Although crime levels are similar to comparable developed nations, the United States is distinctly known to have one of the highest rates of incarceration globally (44). Correctional populations bear a higher burden of the national HIV incidence five times that of the general adult population (45). Through mass incarceration policies, Black persons and their families have been adversely and disproportionately impacted especially in being removed from societal and household settings, and placed in prisons and jails. Adams et al. modeled various intervention strategies aimed to prescribe PrEP to Black women and men affected by mass incarceration and demonstrated that prioritizing Black adults with criminal justice involvement for PrEP delivery can reduce HIV transmission in an urban population (46). Consistent with Adams et al., this study highlights that correctional facilities are likely to be an optimal setting to provide PrEP to those who have been incarcerated or released from jails and prisons (46). Considering the dual impact of HIV and incarceration on Black adults in the United States (47), the delivery of HIV prevention services must be restructured and correctional facilities should be leveraged to reach this key population $(22,48)$.

Beyond the structural impact of mass incarceration on Black adults, in the present study, there were social and individual factors associated with PrEP use, including sexual- and drug-related behaviors. The prevalence of HIV in a concentrated geosocial space has been demonstrated to mediate the influence of social (e.g., drug use) and structural (e.g., incarceration) factors on individual-level behaviors (e.g., sexual activity, multiple sexual partners, and injection drug use), to the extent that interactions with multilevel factors can create varying pathways of HIV vulnerability for Black adults, especially women (49). Through social interactions with people involved in various criminal justice systems, it is possible that individuals with sexual and drug use behaviors are more vulnerable to HIV, which renders greater individual-level protection and, subsequently PrEP engagement among Black adults at

Loading [MathJax]/jax/output/CommonHTML/jax.js reported substance use during sex, only a small proportion had ever 
used PrEP. Given that low perceptions of HIV risk can negatively influence awareness and willingness to use PrEP $(39,42,50)$, low PrEP use in the sample may be explained by a broad lack of HIV risk perception and PrEP awareness. Similarly, social and structural level factors such as medical mistrust $(51,52)$, racial bias by medical professionals $(52)$ and health care service inaccessibility $(20,49)$ may have also limited PrEP use in the present study. Despite the low prevalence of PrEP use, a promising trend emerged in the data. Findings suggest that when Black adults were aware of PrEP, a higher proportion reported ever using the medication. Established correlations denote the influence of sexual partners, correctional institutions, and health care providers on shaping PrEP use outcomes among Black adults. Interventions would benefit from utilizing the social networks and criminal justice systems that Black adults interact with to improve PrEP uptake.

Although the current research contribute to the literature by assessing important sociodemographic and behavioral correlations of PrEP awareness and use among Black adults, this study is not without limitations. First, we were not able to determine the temporal relationship between the correlates and outcome variables. The cross-sectional nature of the data presents challenges with making causal inferences. For example, PrEP users were more likely to report lifetime incarceration, concurrent sexual partners, and substance use during sex; however, we were not able to detect whether PrEP use was initiated prior to or following to the occurrence of these factors. Temporality may be particularly important for assessing the association between PrEP use in Black adults and incarceration to understand: whether incarceration status predicts engagement in PrEP care, and whether care continues in the period upon incarceration release. Future research should analyze this association using longitudinal methods to gain greater insights into the temporal relationship among these factors. Secondly, data were based on self-report measures, which may render concern for potential recall and social desirability biases. Although surveys were selfadministered, participants may have underreported behaviors given the highly sensitive nature of some survey items. Lastly, study findings may not be generalizable to more diverse groups of Black adults.

In the absence of widespread PrEP awareness and use among Black adults, HIV prevention efforts are faced with significant challenges to ending the epidemic in the United States. The current study advances the emerging literature by documenting multiple factors associated with PrEP outcomes among this key HIV-prevention target group. Our findings highlight that specific individual, social and structural factors are correlated with PrEP awareness (i.e., incarceration history and STI testing) and use (i.e., incarceration history, concurrent sexual partners and substance use during sex). Results point to optimizing sexual health and drug rehabilitation services offered in correctional settings as a potential pathway to inform individuals about HIV risk behaviors and facilitate PrEP access among Black adults. Given the complex interplay between structural and interpersonal factors on PrEP outcomes, a culturally-comprehensive public policy is necessary to better facilitate access to HIV prevention services, especially for substance-using adults with criminal justice involvement. Doing so would serve the individual and their community by controlling HIV transmission in the United States. Future research should further explore the multilevel factors correlated with PrEP awareness and use to identify additional areas for targeted uptake.

\section{Declarations}

Funding: This study was funded by Les Brun Pilot Funding, Buffalo Center for Social Research.

Conflicts of interest/Competing interests: The authors have no relevant financial or non-financial interests to disclose.

Loading [MathJax]/jax/output/CommonHTML/jax.js 
Ethics approval: This study was performed in line with the principles of the Declaration of Helsinki. Approval was granted by the University at Buffalo's Institutional Review Board (May 2018).

Consent to participate: Informed consent was obtained from all individual participants included in the study.

Consent for publication: The authors affirm that human research participants provided informed consent for publication of the data located in Table 1, Table 2, and Table 3.

Availability of data and material: The dataset analyzed during the current study are available from the senior author on reasonable request.

\section{Code availability: N/A}

Authors' contributions: Conceptualization: Noelle St. Vil, Sarahmona Przybyla and Natalie Leblanc; Methodology: Noelle St. Vil; Formal analysis and investigation: Kennethea Wilson, Sarahmona Przybyla, Jacob Bleasdale and Steven Gabriel; Writing-original draft preparation: Kennethea Wilson, Jacob Bleasdale and Steven Gabriel; Writingreview and editing: Kennethea Wilson, Noelle St. Vil, Sarahmona Przybyla, Jacob Bleasdale, Steven Gabriel, and Natalie Leblanc; Funding acquisition: Noelle St. Vil; Resources: Noelle St. Vil; Supervision: Sarahmona Przybyla

\section{References}

1. Centers for Disease Control and Prevention. Estimated HIV incidence and prevalence in the United States, 2014-2018. 2020.

2. United States Census Bureau. American community survey. 2019.

3. Centers for Disease Control and Prevention. Diagnoses of HIV infection in the United States and dependent areas, 2018 (preliminary). National Center for HIV/AIDS VH, STD, and TB Prevention; 2019.

4. Centers for Disease Control and Prevention. Lifetime risk of HIV diagnosis. 2016.

5. Kaiser Family Foundation. HIV testing in the United States 2019 [Available from: https://www.kff.org/hivaids/fact-sheet/hiv-testing-in-the-united-states/].

6. Centers for Disease Control and Prevention. HIV and African American People. 2021.

7. Phillips G 2nd, McCuskey D, Ruprecht MM, Curry CW, Felt D. Structural interventions for HIV prevention and care among US men who have sex with men: a systematic review of evidence, gaps, and future priorities. AIDS Behav. 2021:1-13.

8. Centers for Disease Control and Prevention. Sexually transmitted disease surveillance 2018. Atlanta: U.S. Department of Health and Human Services; 2019.

9. Beatty LA, Wheeler D, Gaiter J. HIV prevention research for African Americans: current and future directions. Journal of Black Psychology. 2004;30(1):40-58.

10. Williams JK, Wyatt GE, Wingood G. The four Cs of HIV prevention with African Americans: crisis, condoms, culture, and community. Curr HIV/AIDS Rep. 2010;7(4):185-93.

11. Centers for Disease Control and Prevention. CDC statement on FDA approval of drug for HIV prevention. 2012.

12. United States Public Health Service. Preexposure prophylaxis for the prevention of HIV infection in the United State - 2017 update: clinical practice guideline. 2018.

13. Centers for Disease Control and Prevention. Preexposure prophylaxis for the prevention of HIV in the United e guideline. US Public Health Service; 2018. 
14. United States Department of Health \& Human Services. Ending the HIV epidemic: a plan for America. 2020.

15. Bond KT, Gunn A, Williams P, Leonard NR. Using an intersectional framework to understand the challenges of adopting pre-exposure prophylaxis (PrEP) among young adult Black women. Sex Res Soc Policy. 2021:14.

16. Kanny D, Jeffries WLt, Chapin-Bardales J, Denning P, Cha S, Finlayson T, et al. Racial/ethnic disparities in HIV preexposure prophylaxis among men who have sex with men - 23 urban areas, 2017. MMWR Morb Mortal Wkly Rep. 2019;68(37):801-6.

17. Smith DK, Van Handel M, Grey J. Estimates of adults with indications for HIV pre-exposure prophylaxis by jurisdiction, transmission risk group, and race/ethnicity, United States, 2015. Ann Epidemiol. 2018;28(12):8507.e9.

18. Huang YA, Zhu W, Smith DK, Harris N, Hoover KW. HIV preexposure prophylaxis, by race and ethnicity - United States, 2014-2016. MMWR Morb Mortal Wkly Rep. 2018;67(41):1147-50.

19. Goedel WC, Bessey S, Lurie MN, Biello KB, Sullivan PS, Nunn AS, et al. Projecting the impact of equity-based preexposure prophylaxis implementation on racial disparities in HIV incidence among MSM. Aids. 2020;34(10):1509-17.

20. Johnson AK, Fletcher FE, Ott E, Wishart M, Friedman EE, Terlikowski J, et al. Awareness and intent to use preexposure prophylaxis (PrEP) among African American women in a family planning clinic. J Racial Ethn Health Disparities. 2020;7(3):550-4.

21. Wood S, Gross R, Shea JA, Bauermeister JA, Franklin J, Petsis D, et al. Barriers and facilitators of PrEP adherence for young men and transgender women of color. AIDS Behav. 2019;23(10):2719-29.

22. Ojikutu BO, Bogart LM, Higgins-Biddle M, Dale SK, Allen W, Dominique T, et al. Facilitators and barriers to preexposure prophylaxis (PrEP) use among Black individuals in the United States: results from the National Survey on HIV in the Black Community (NSHBC). AIDS Behav. 2018;22(11):3576-87.

23. Lelutiu-Weinberger C, Wilton L, Koblin BA, Hoover DR, Hirshfield S, Chiasson MA, et al. The role of social support in HIV testing and PrEP awareness among young Black men and transgender women who have sex with men or transgender women. J Urban Health. 2020;97(5):715-27.

24. Ezennia O, Geter A, Smith DK. The PrEP care continuum and Black men who have sex with men: a scoping review of published data on awareness, uptake, adherence, and retention in PrEP care. AIDS Behav. 2019;23(10):2654-73.

25. Auerbach JD, Kinsky S, Brown G. Charles V. Knowledge, attitudes, and likelihood of pre-exposure prophylaxis (PrEP) use among US women at risk of acquiring HIV. AIDS Patient Care STDS. 2015;29(2):102-10.

26. Calabrese SK, Earnshaw VA, Underhill K, Hansen NB, Dovidio JF. The impact of patient race on clinical decisions related to prescribing HIV pre-exposure prophylaxis (PrEP): assumptions about sexual risk compensation and implications for access. AIDS Behav. 2014;18(2):226-40.

27. Tomko C, Park JN, Allen ST, Glick J, Galai N, Decker MR, et al. Awareness and interest in HIV pre-exposure prophylaxis among street-based female sex workers: results from a US context. AIDS Patient Care STDS. 2019;33(2):49-57.

28. Philbin MM, Parker CM, Parker RG, Wilson PA, Garcia J, Hirsch JS. The promise of pre-exposure prophylaxis for Black men who have sex with men: an ecological approach to attitudes, beliefs, and barriers. AIDS Patient Care STDS. 2016;30(6):282-90.

29. Friedman MR, Sang JM, Bukowski LA, Chandler CJ, Egan JE, Eaton LA, et al. Prevalence and correlates of PrEP awareness and use among Black men who have sex with men and women (MSMW) in the United States. AIDS

Loading [MathJax]/jax/output/CommonHTML/jax.js 
30. Eaton LA, Matthews DD, Driffin DD, Bukowski L, Wilson PA, Stall RD. A multi-US city assessment of awareness and uptake of pre-exposure prophylaxis (PrEP) for HIV prevention among Black men and transgender women who have sex with men. Prev Sci. 2017;18(5):505-16.

31. St Vil NM, Leblanc NM, Giles KN. The who and why of consensual nonmonogamy among African Americans. Arch Sex Behav. 2020.

32. Aaron E, Blum C, Seidman D, Hoyt MJ, Simone J, Sullivan M, et al. Optimizing delivery of HIV preexposure prophylaxis for women in the United States. AIDS Patient Care STDS. 2018;32(1):16-23.

33. Edwards GG, Reback CJ, Cunningham WE, Hilliard CL, McWells C, Mukherjee S, et al. Mobile-enhanced prevention support study for men who have sex with men and transgender women leaving jail: protocol for a randomized controlled trial. JMIR Res Protoc. 2020;9(9):e18106.

34. Harawa NT, Brewer R, Buckman V, Ramani S, Khanna A, Fujimoto K, et al. HIV, sexually transmitted infection, and substance use continuum of care interventions among criminal justice-involved Black men who have sex with men: a systematic review. Am J Public Health. 2018;108(S4):e1-9.

35. Severe M, Scheidell JD, Dyer TV, Brewer RA, Negri A, Turpin RE, et al. Lifetime burden of incarceration and violence, internalized homophobia, and HIV/STI risk among Black men who have sex with men in the HPTN 061 study. AIDS Behav. 2020.

36. Centers for Disease Control and Prevention. Sexually transmitted infections prevalence, incidence, and cost estimates in the United States 2021 [Available from: https://www.cdc.gov/std/statistics/prevalence-2020-at-aglance.htm].

37. National Commission on Correctional Health Care. STI testing for adolescents and adults upon admission to correctional facilities: National Commission on Correctional Health Care; 2020 [Available from: https://www.ncchc.org/STI-testing-upon-admission].

38. Centers for Disease Control and Prevention. 2015 sexually transmitted diseases treatment guidelines. 2015.

39. Dauria EF, Levine A, Hill SV, Tolou-Shams M, Christopoulos K. Multilevel factors shaping awareness of and attitudes toward pre-exposure prophylaxis for HIV prevention among criminal justice-involved women. Arch Sex Behav. 2020.

40. Brinkley-Rubinstein L, Dauria E, Tolou-Shams M, Christopoulos K, Chan PA, Beckwith CG, et al. The path to implementation of HIV pre-exposure prophylaxis for people involved in criminal justice systems. Curr HIV/AIDS Rep. 2018;15(2):93-5.

41. Ramsey SE, Ames EG, Brinkley-Rubinstein L, Teitelman AM, Clarke J, Kaplan C. Linking women experiencing incarceration to community-based HIV pre-exposure prophylaxis care: protocol of a pilot trial. Addict Sci Clin Pract. 2019;14(1):8.

42. Peterson M, Macmadu A, Truong AQ, Rich J, Pognon K, Lurie M, et al. Pre-exposure prophylaxis awareness and interest among participants in a medications for addiction treatment program in a unified jail and prison setting in Rhode Island. J Subst Abuse Treat. 2019;106:73-8.

43. Collier KL, Colarossi LG, Sanders K. Raising awareness of pre-exposure prophylaxis (PrEP) among women in New York City: community and provider perspectives. J Health Commun. 2017;22(3):183-9.

44. Walmsley R. World prison population list: National Institute of Corrections; 2015 [11:[Available from: https://nicic.gov/world-prison-population-listeleventh-

edition\#: :text=Seychelles\%20has\%20the\%20highest\%20prison,lower\%20than\%20150\%20per\%20100\%2C000].

45. Valera P, Chang Y, Lian Z. HIV risk inside U.S. prisons: a systematic review of risk reduction interventions Loading [MathJax]/jax/output/CommonHTML/jax.js 017;29(8):943-52. 
46. Adams JW, Khan MR, Bessey SE, Friedman SR, McMahon JM, Lurie MN, et al. Preexposure prophylaxis strategies for African-American women affected by mass incarceration. Aids. 2021;35(3):453-62.

47. Wohl DA, Rosen D, Kaplan AH. HIV and incarceration: dual epidemics. AIDS Read. 2006;16(5):247-50, 57-60.

48. Rowell-Cunsolo TL, Szeto B, Sampong SA, Larson EL. Predictors of sexual behaviour among men and women in New York City area prisons. Cult Health Sex. 2016;18(12):1393-406.

49. Brawner BM. A multilevel understanding of HIV/AIDS disease burden among African American women. J Obstet Gynecol Neonatal Nurs. 2014;43(5):633. :- 43; quiz E49-50..

50. Przybyla SM, Cerulli C, Bleasdale J, Wilson K, Hordes M, El-Bassel N, et al. "I think everybody should take it if they're doing drugs, doing heroin, or having sex for money": a qualitative study exploring perceptions of preexposure prophylaxis among female participants in an opioid intervention court program. Subst Abuse Treat Prev Policy. 2020;15(1):89.

51. Tekeste M, Hull S, Dovidio JF, Safon CB, Blackstock O, Taggart T, et al. Differences in medical mistrust between Black and White women: implications for patient-provider communication about PrEP. AIDS Behav. 2019;23(7):1737-48.

52. Quinn K, Dickson-Gomez J, Zarwell M, Pearson B, Lewis M. "A gay man and a doctor are just like, a recipe for destruction": how racism and homonegativity in healthcare settings influence PrEP uptake among young Black MSM. AIDS Behav. 2019;23(7):1951-63.

\section{Tables}


Table I. Sample characteristics $(\mathrm{N}=666)$

\begin{tabular}{|c|c|}
\hline & $\mathrm{n}(\%)$ \\
\hline \multicolumn{2}{|l|}{ Gender } \\
\hline Male & $203(30.53)$ \\
\hline Female & $462(69.47)$ \\
\hline \multicolumn{2}{|l|}{ Missing $=1$} \\
\hline \multicolumn{2}{|l|}{ Age } \\
\hline 18-29 years & $355(53.30)$ \\
\hline $30-40$ years & $311(46.70)$ \\
\hline \multicolumn{2}{|l|}{ Education } \\
\hline Less than high school & $159(23.87)$ \\
\hline Greater than high school & 507 (76.13) \\
\hline History of incarceration & $77(11.60)$ \\
\hline \multicolumn{2}{|l|}{ Missing $=2$} \\
\hline \multicolumn{2}{|l|}{ Income } \\
\hline 12,000 & $91(13.66)$ \\
\hline 12,000 & $575(86.34)$ \\
\hline Monogamous relationship & $489(73.42)$ \\
\hline \multicolumn{2}{|l|}{ Sexual orientation } \\
\hline Heterosexual & $521(84.58)$ \\
\hline Lesbian/gay/bisexual & $95(15.42)$ \\
\hline \multicolumn{2}{|l|}{ Missing $=50$} \\
\hline Substance use (e.g., alcohol, marijuana) during sex & $391(58.71)$ \\
\hline Lifetime STI testing & $405(60.81)$ \\
\hline Lifetime STI diagnosis $(\mathrm{n}=405)$ & $158(39.01)$ \\
\hline Number of current sex partners & $2.53(9.43)^{\mathrm{a}}$ \\
\hline \multicolumn{2}{|l|}{ Missing $=16$} \\
\hline Number of lifetime sex partners & $12.18(44.4)^{\mathrm{a}}$ \\
\hline \multicolumn{2}{|l|}{ Missing $=26$} \\
\hline PrEP aware & $192(28.82)$ \\
\hline Lifetime PrEP use $(n=192)$ & $39(20.31)$ \\
\hline
\end{tabular}

a Mean [SD]

Table II. Adjusted odds ratios (aOR) from multivariable logistic regression model assessing associations between PrEP awareness and covariates $(\mathrm{N}=614)$

\begin{tabular}{lll}
\hline Outcome & Covariate & aOR (95\% CI) \\
\hline PrEP Awareness & & $1.73(1.04,2.90)^{\mathrm{a}}$ \\
& Incarceration history & $1.49(0.81,2.75)$ \\
& Income & $1.76(1.19,2.59)^{\mathrm{a}}$ \\
& Lifetime STI testing & $1.43(0.93,2.21)$ \\
& Monogamous relationship & $1.28(0.86,2.21)$ \\
& Sexual orientation & $1.38(0.95,2.00)$ \\
\hline
\end{tabular}

a Significance at $p<0.05$ 
Table III. Adjusted odds ratios (aOR) from multivariable logistic regression model assessing associations between PrEP use and covariates $(\mathrm{N}=132)$

\begin{tabular}{lll}
\hline Outcome & Covariate & aOR (95\% CI $)$ \\
\hline Lifetime PrEP Use & & \\
& Age & $0.34(0.11,1.03)$ \\
\hline & Current sex partners & $1.09(1.00,1.18)^{\mathrm{a}}$ \\
\hline & Education & $0.32(0.09,1.08)$ \\
\hline Gender & $1.34(0.40,4.46)$ \\
\hline Incarceration history & $9.96(2.82,35.14)^{\mathrm{a}}$ \\
\hline & Lifetime STI diagnosis & $1.32(0.44,3.89)$ \\
\hline
\end{tabular}

\footnotetext{
${ }^{a}$ Significance at $p<0.05$
} 